\title{
Dexmedetomidine may upregulate the expression of caveolin-1 in lung tissues of rats with sepsis and improve the short-term outcome
}

\author{
YUN ZHANG ${ }^{1}, \mathrm{KE} \mathrm{RAN}^{1}$, SHU-BIN ZHANG $^{2}$, LILI JIANG $^{3}$, DAN WANG $^{1}$ and ZHI-JIAN LI ${ }^{1}$ \\ ${ }^{1}$ Department of Anesthesiology, The Second XiangYa Hospital of Central South University, Changsha, Hunan 410011; \\ ${ }^{2}$ Department of Cell Biology, School of Life Science, Central South University, Changsha, Hunan 410013; \\ ${ }^{3}$ Department of Anesthesiology, Affiliated Hospital of Qingdao University, Qingdao, Shandong 266005, P.R. China
}

Received November 7, 2015; Accepted November 10, 2016

DOI: $10.3892 / \mathrm{mmr} .2016 .6050$

\begin{abstract}
Dexmedetomidine (DXM) is a selective $\alpha 2$-adrenoceptor ( $\alpha 2$-AR) and imidazoline receptor (IR) agonist that has been reported to regulate inflammatory responses mediated by diverse signaling pathways through $\alpha 2-\mathrm{AR}$. The majority of the reported receptors or downstream molecules have been demonstrated to locate with caveolin-1, a protein suggested to participate in regulating Toll-like receptor 4 (TLR4)-mediated inflammatory responses and the pathogen endocytosis capability of macrophages. The present study hypothesized that DXM may influence these pathways by regulating the expression of caveolin-1 and mediating the subsequent effects. Using a cecal-ligation and puncture-induced rat sepsis model, it was initially observed that pre-emptive DXM is able to upregulate and stabilize the amount of caveolin-1 expression, which may be partly antagonized by both $\alpha 2-\mathrm{AR}$ and the IR antagonist atepamezole (APZ). The pathophysiological parameters indicated that DXM is able to inhibit secondary lung injury, in addition to the rise of body temperature and arterial lactate accumulation, however it marginally increased arterial glucose and the murine sepsis score, which can be largely antagonized by APZ. The overall effect was beneficial and improved the 24-h cumulative survival rate of rats with sepsis. In conclusion, preemptive clinical sedative doses of DXM may upregulate the expression of caveolin-1 downregulated by sepsis, which may contribute to the inhibition of inflammatory pathways such as TLR4-mediated pathways. Furthermore, DXM may favor the improvement of short-term outcomes by the regulation of other metabolic pathways.
\end{abstract}

Correspondence to: Dr Zhi-Jian Li, Department of Anesthesiology, The Second XiangYa Hospital of Central South University, Furong, 139 Ren-min Road, Changsha, Hunan 410011, P.R. China E-mail: 1104950322@qq.com

Key words: dexmedetomidine, caveolin-1, sepsis, inflammation, secondary lung injury, signaling crosstalk

\section{Introduction}

Sepsis is a systemic inflammatory response syndrome with a proven or suspected infectious etiology (1). The early stages of sepsis are classically characterized by fever and blood hyperdynamics, with rapidly developing secondary organ injury, and hypothermia and death as sepsis progresses $(1,2)$. The mortality rate of sepsis remains high and the therapy remains intractable.

Dexmedetomidine (DXM) is a selective $\alpha 2$-adrenoceptor $(\alpha 2-\mathrm{AR})$ and imidazoline receptor (IR) agonist (3), which was formally approved for sedation and adjunct analgesia for patients in the intensive care unit (ICU) by the US Food and Drug Administration in 1999, particularly recommended for patients with sepsis (4) Clinical studies of patients with severe sepsis and other critically ill patients identified that DXM may marginally improve the survival rate and significantly inhibit the release of pro-inflammatory factors in patients with sepsis (5-7). These beneficial effects were confirmed by an animal study (8), however the underlying signaling pathways reported from different animal and cytological models presented a wide range of pathways including: Toll-like receptor 4 (TLR4)/myeloid differentiation primary response gene 88 (MyD88)/nuclear factor- $\kappa \mathrm{B}(\mathrm{NF}-\kappa \mathrm{B})$ or mitogen-activated protein kinase [c-Jun N-terminal kinase, extracellular signal-regulated kinases $($ ERK)1/2] $(8,9)$, endothelial nitric oxide synthase (eNOS)/nitric oxide (10) and Janus kinase/signal transducers and activators of transcription (11). How DXM can affect so numerous signaling pathways or downstream molecules remains unclear.

Caveolae, typically flask-shaped membrane invaginations expressed in various cell types, are abundant in lung, muscle and adipose tissues, and have been demonstrated to participate in the regulation of lipid and glucose metabolism (12), in addition to the maturation of immunocytes and inflammation responses (13). Caveolin-1 is a protein that maintains the morphological and functional integrity of caveolae (12). Knockout of caveolin-1 decreases the survival rate in mice with cecal ligation and puncture (CLP)-induced sepsis (14). Jiao et al (15) identified that tyrosine (Tyr) 14 phosphorylation of caveolin-1 induced interaction with TLR4 and mediated 
TLR4/MyD88 signaling regulation. Additional studies have demonstrated that receptors including G-protein coupled receptors (GPCRs), $\alpha$-ARs, $\beta$-ARs (16), tumor necrosis factor receptor (17) and downstream molecules including $\mathrm{G}_{\alpha}$ subunits (Gs, Gi and Gq) (16), eNOS (18) and ERK1/2 (19), localized to the caveolae. GPCR activation led to the release and translocation of G $\beta \gamma$ subunits and subsequent Src-dependent Tyr phosphorylation of caveolin-1 (20). Following these studies, it was hypothesized that DXM may affect inflammatory pathways through the influence on caveolin-1.

The present study aimed to evaluate the effect of DXM on the most frequent clinical manifestations of sepsis and the expression of caveolin-1 in lung tissues, and to identify the association between inflammatory pathways and the characteristic receptors of DXM.

\section{Materials and methods}

Animals. A total of 170 male Sprague-Dawley rats aged 8 weeks (weight, 250-300 g) were obtained from the Animal Center of the Second XiangYa Hospital of Central South University (Changsha, China) and housed in the Animal Laboratory Center of the Second XiangYa Hospital of Central South University, in groups of three per cage. All rats were weighed and numbered on arrival and acclimated for 7 days, under $24^{\circ} \mathrm{C}$ and $48 \%$ humidity, a 12-h light/dark circadian cycle, and were provided with specific pathogen-free rodent diet and water ad libitum throughout all experiments. Animals were euthanized with an overdose of pentobarbital injected after the experiments. All animal procedures were approved by the Animal Care and Use Committee of XiangYa Medical College, Central South University (Changsha, China).

Materials. The small-animal-specialized digital thermometer (AT320; Da-Xiong Ltd., Shenzhen, China) and blood gas analyzer (GEM Premier 3000; Instrumentation Laboratory Co., Bedford, MA, USA) were used. The silk suture (Ethilon; Ethicon, Inc., Somerville, NJ, USA), sodium dodecyl sulfate (SDS) buffer and phosphate-buffered saline (PBS; Beijing Dingguo Changsheng Biotechnology Co., Ltd., Beijing, China) were donated by Dr Lili Jiang (Department of Anesthesiology, Affiliated Hospital of Qingdao University, Qingdao, China). DXM and ketamine (HengRui Medicine, Ltd., Nanjing, China) were purchased from Second XiangYa Hospital of Central South University. Pentobarbital sodium (Sigma-Aldrich; Merck Millipore, Darmstadt., Germany), atepamezole (APZ; Axon Medchem, Groningen, Netherlands), D46G3 ${ }^{\mathrm{XP}}$ caveolin-1 rabbit monoclonal antibody [horseradish peroxidase (HRP)-conjugated; 1:10,000; catalog no. 3267S] and 13E5 $\beta$-actin rabbit monoclonal $(1: 1,000$; catalog no. 4970S) antibodies (Cell Signaling Technology, Inc., Danvers, MA, USA) were purchased from respective companies. The HRP-conjugated secondary antibody (1:1,000; catalog no. CW0103M) was obtained from Beijing ComWin Biotech Co., Ltd. (Beijing, China). Enhanced bicinchoninic acid assay (BCA) protein assay kit was purchased from Beyotime Institute of Biotechnology (Shanghai, China).

CLP surgery. All rats were anesthetized with pentobarbital sodium [45 mg/kg, intra-peritoneal (i.p.)] and ketamine
(1.5 mg/100 g, intravenous). Following a previous study (21), a severe polymicrobial septic model was induced by CLP. Rats were fixed on a surgical board following the shaving of abdominal hair. Surgical fields were covered with sterile drapes after disinfection with iodine complex (three times), then a $1-2 \mathrm{~cm}$ lower-midline laparotomy was conducted. The cecum was exposed and ligated tightly $25 \%$ distal to the ileocecal valve with a $6-0$ silk suture, and perforated twice with an 18-gauge needle on the same side near and distal to the ligation, respectively. The cecum was squeezed gently to extrude two small amounts of feces after which the cecum was returned to abdominal cavity. The abdomen was closed with 3-0 silk sutures in two layers. Then $5 \mathrm{ml} / 100 \mathrm{~g} 37^{\circ} \mathrm{C}$ normal saline was injected subcutaneously using a $25 \mathrm{G}$ needle prior to the rat being transferred to a recovery cage. Perioperative body temperature was protected with temperature-adjusting blankets and monitored at $1 \mathrm{~h}$ intervals.

Experimental protocol. Rats were randomly divided into five groups: Control group (Control); sham surgery (Sham); CLP (CLP); DXM + CLP (DXM); and DXM + APZ + CLP (APZ). Control rats did not undergo any procedures. Sham surgery was conducted with laparotomy and cecum exposure without ligation and puncture. CLP was conducted as aforementioned. DXM $(2 \mu \mathrm{g} / 100 \mathrm{~g}, 10 \mu \mathrm{g} / \mathrm{ml})$ or an equal volume normal saline was injected i.p. $10 \mathrm{~min}$ prior to surgery. APZ $(25 \mu \mathrm{g} / 100 \mathrm{~g}$, $1 \mathrm{mg} / \mathrm{ml}$ ) or an equal volume normal saline was injected i.p. simultaneously with pentobarbital injection.

Survival rate, anal temperature and disease severity monitoring, and arterial blood gas $(A B G)$ analysis. A total of 65 rats were randomly divided into five groups: Control and Sham (10 per group), CLP, DXM and APZ (15 per group) as aforementioned. All rats were monitored up to $24 \mathrm{~h}$ or until death, and the time when an animal died was recorded. Left ventricular blood was collected when rats died or were euthanized at $24 \mathrm{~h}$ for ABG. Anal temperature and disease severity were determined at pre-set time-points (immediately prior to surgery, 4, 8, 12 and 24 h postoperatively) with a digital thermometer and a scoring system, murine sepsis score (MSS), developed by Shrum et al (22). The criteria in MSS (data not shown) include: Degree of piloerection, spontaneous activity, response to stimuli, level of consciousness, openness of eyes, posture and degree of labored breathing. Each criterion was scored from 0 to 4 and a total MSS score was calculated. Mortality rises as MSS increases.

Lung tissue collection. At each time-point (immediately prior to surgery, 4, 8, 12 and 24 h postoperatively), thoracotomy was performed on the rats (three rats per group in control and sham groups, five per group in the rest of the groups), with pulmonary transfusion of $0.9 \%$ normal saline under speed of $120 \mathrm{ml} / \mathrm{h}$ for $15 \mathrm{~min}$ with drainage of the blood from left atrium. Then both lungs were removed. The lower and middle lobes of right lung tissues were snap frozen in the liquid nitrogen and stored at $-80^{\circ} \mathrm{C}$ for subsequent protein detection, and the whole left lung was perfused with $1.5 \mathrm{ml}$ $10 \%$ formalin via the bronchus and stored in $10 \%$ formalin for hematoxylin and eosin (H\&E) staining and pathological analyses. 
Western blot analysis. Frozen rat lung tissues were homogenized and the lysates were prepared in ice-cold lysis buffer and centrifuged $\left(10,000 \mathrm{x} \mathrm{g},-4^{\circ} \mathrm{C}, 10 \mathrm{~min}\right)$. The supernatant was collected and normalized for equal amounts of total protein measured with the BCA method; $60 \mu \mathrm{g}$ protein from each sample were separated on a $12 \%$ SDS-polyacrylamide gel (SDS-PAGE) and transferred to PVDF membranes (Merck Millipore). The membranes were blocked with 5\% non-fat milk and incubated overnight with the primary anti-caveolin-1 antibody at $4^{\circ} \mathrm{C}$, followed by incubation with the HRP-conjugated secondary antibody for $4 \mathrm{~h}$. Cellular $\beta$-actin protein was immunodetected as the internal standard.

HE staining and histological injury scoring for lung tissues. Formalin-fixed lungs were embedded in paraffin and serially sectioned in toto, then stained with H\&E. A total of five images per slide were captured with a microscope (Nikon Eclipse E200; Nikon Corporation, Tokyo, Japan) at magnifications of $x 4$ and $x 40$. Histological changes, including alveolar wall edema, congestion, hemorrhage and inflammatory cell infiltration under a magnification of $\mathrm{x} 40$, were scored by a pathologist blinded to the present study, as previously described (8). Each criterion was scored between 0 (normal) and 5 (severe), and the overall pulmonary inflammation was categorized according to the sum of the score $(0-5$, normal to minimal inflammation; 6-10, mild inflammation; 11-15, moderate inflammation; 16-20, severe inflammation).

Statistical analysis. Data were presented as the mean \pm standard deviation and analyzed with SPSS software, version 20.0 (SPSS, Inc., Chicago, IL, USA). Differences between groups were determined by analysis of variance, followed by a post hoc test (least significant difference method; LSD) in ABG and histological scoring data. The missing values of MSS and anal temperature due to mortality were interpolated with the maximal MSS score and the temperature measured immediately prior to mortality, respectively. Western blotting images were read and calculated with Image J2X software (http://imagej.net/ImageJ2). Then MSS, anal temperature and caveolin-1 expression data were analyzed with repeated analysis of variance, followed by post hoc analysis (LSD). The cumulative survival rates among groups were analyzed with log-rank $\chi^{2}$ test and the survival curve with the Kaplan-Meier method. $\mathrm{P}<0.05$ was considered to indicate a statistically significant difference.

\section{Results}

Survival rate. As demonstrated in Fig. 1, the survival rate 24-h post-operatively was $100,90,20,40$ and 33.3, respectively, for the Control, Sham, CLP, DXM and APZ groups. No significant differences were identified between the Control and Sham groups. CLP surgery markedly decreased survival rate compared with the Sham group, and survival rate was notably improved by pre-emptive DXM treatment. The antagonism of APZ did not significantly decrease the protection of DXM.

The severity of disease. The MSS score, which was reported to be highly predictive of sepsis progression and mortality and was paralleled with systemic pro-inflammatory factors in

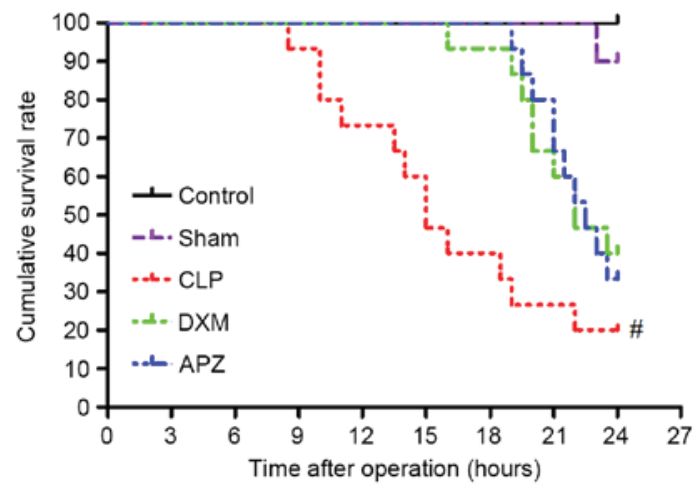

Figure 1. The effect of DXM and its antagonist APZ on survival rates of septic rats induced by CLP. Control, control group $(n=10)$; Sham, sham operation group ( $n=10)$; CLP, CLP operation group $(n=15)$; DXM, DXM pretreatment group $(n=15)$; APZ, APZ antagonizing group $(n=15)$. The survival rate $24 \mathrm{~h}$ after operation was analyzed. " $\mathrm{P}<0.05$, vs. Sham, DXM and APZ groups, respectively. DXM, dexmedetomidine; APZ, atepamezole; CLP, cecal ligation and puncture.

CLP-induced mice models (22), was employed to evaluate the effect of DXM. Fig. 2A provides the MSS scoring at pre-set time-points after surgery from individual rats in different treatment groups without missing data interpolation. Control rats scored 0 at all time-points. Sham-operated rats scored similar to CLP-operated rats $4 \mathrm{~h}$ postoperatively and went back to control level $8 \mathrm{~h}$ postoperatively, with the exception of one whose cecum was in the right upper quadrant and kept high scores after surgery. Scores of CLP-operated rats increased continuously $8 \mathrm{~h}$ postoperatively. DXM pretreatment resulted in the rise of scoring, however inhibited the rising speed $4 \mathrm{~h}$ postoperatively compared with that of CLP; however, APZ partly antagonized this effect. Fig. 2B demonstrates the differences of MSS among groups with missing data interpolation. MSS scores significantly increased after Sham and CLP surgery and only returned to the control level $8 \mathrm{~h}$ after surgery in the Sham group. DXM markedly increased scores at $4 \mathrm{~h}$ and maintained a similar level until $12 \mathrm{~h}$ postoperatively compared with the CLP group. APZ significantly antagonized the effect of DXM and reduced the scores until $24 \mathrm{~h}$ postoperatively.

$A B G$ analysis. As demonstrated in Table I, there was no significant difference of $\mathrm{pH}$ among the groups. Blood lactate and base excess significantly increased in the CLP group compared with the Sham group, DXM marginally ameliorated lactate accumulation, and APZ marginally antagonized these effects, however these were not significant. Blood glucose (Glu) significantly increased in the Sham, CLP and DXM groups, however remained higher in the DXM group compared with the CLP group but (not statistically significant). Glu was markedly antagonized by APZ.

Anal temperature. Fig. 3A demonstrates the anal temperature $\left({ }^{\circ} \mathrm{C}\right)$ of individual rats at pre-set time-points after surgery from different treatment groups without missing data interpolation. Sham-operated rats convergently maintained a higher temperature without influence on short-term survival. CLP surgery affected the temperature and survival of rats. DXM and APZ effectively improved survival rate, however DXM significantly inhibited the increase of temperature as sepsis 
Table I. Arterial blood gas analysis data.

\begin{tabular}{lcccr}
\hline Group & $\mathrm{pH}$ & Lac $(\mathrm{mmol} / \mathrm{ml})$ & $\mathrm{BE}(\mathrm{mmol} / \mathrm{ml})$ & $\mathrm{Glu}(\mathrm{mmol} / \mathrm{ml})$ \\
\hline Control & $7.42 \pm 0.08$ & $1.34 \pm 0.45$ & $0.33 \pm 2.77$ & $6.40 \pm 1.76$ \\
Sham & $7.40 \pm 0.08$ & $1.79 \pm 0.33^{\mathrm{a}}$ & $-1.45 \pm 3.31^{\mathrm{a}}$ & $9.85 \pm 2.31$ \\
CLP & $7.32 \pm 0.13$ & $4.5 \pm 1.35$ & $-4.18 \pm 5.04$ & $10.85 \pm 2.51$ \\
DXM & $7.45 \pm 0.13$ & $3.58 \pm 1.01$ & $-4.10 \pm 4.78$ & $12.67 \pm 5.74$ \\
APZ & $7.43 \pm 0.14$ & $4.02 \pm 0.89$ & $-3.96 \pm 3.56$ & $7.59 \pm 1.86^{\mathrm{b}}$ \\
\hline
\end{tabular}

Control, control group ( $\mathrm{n}=10)$; Sham, sham operation group $(\mathrm{n}=10)$; CLP, CLP operation group ( $\mathrm{n}=15)$; DXM, DXM treatment group ( $\mathrm{n}=15)$; APZ, APZ antagonizing group $(n=15)$. The femoral blood collected when rats died naturally or were euthanized at $24 \mathrm{~h}$ were determined immediately after death and were analyzed. ${ }^{\mathrm{P}} \mathrm{P}<0.001$, vs. CLP group; ${ }^{\mathrm{P}} \mathrm{P}<0.05$, vs. DXM group. Lac, blood lactate; BE, base excess; Glu, blood glucose; CLP, cecal ligation and puncture; DXM, dexmedetomidine; APZ, atepamezole.
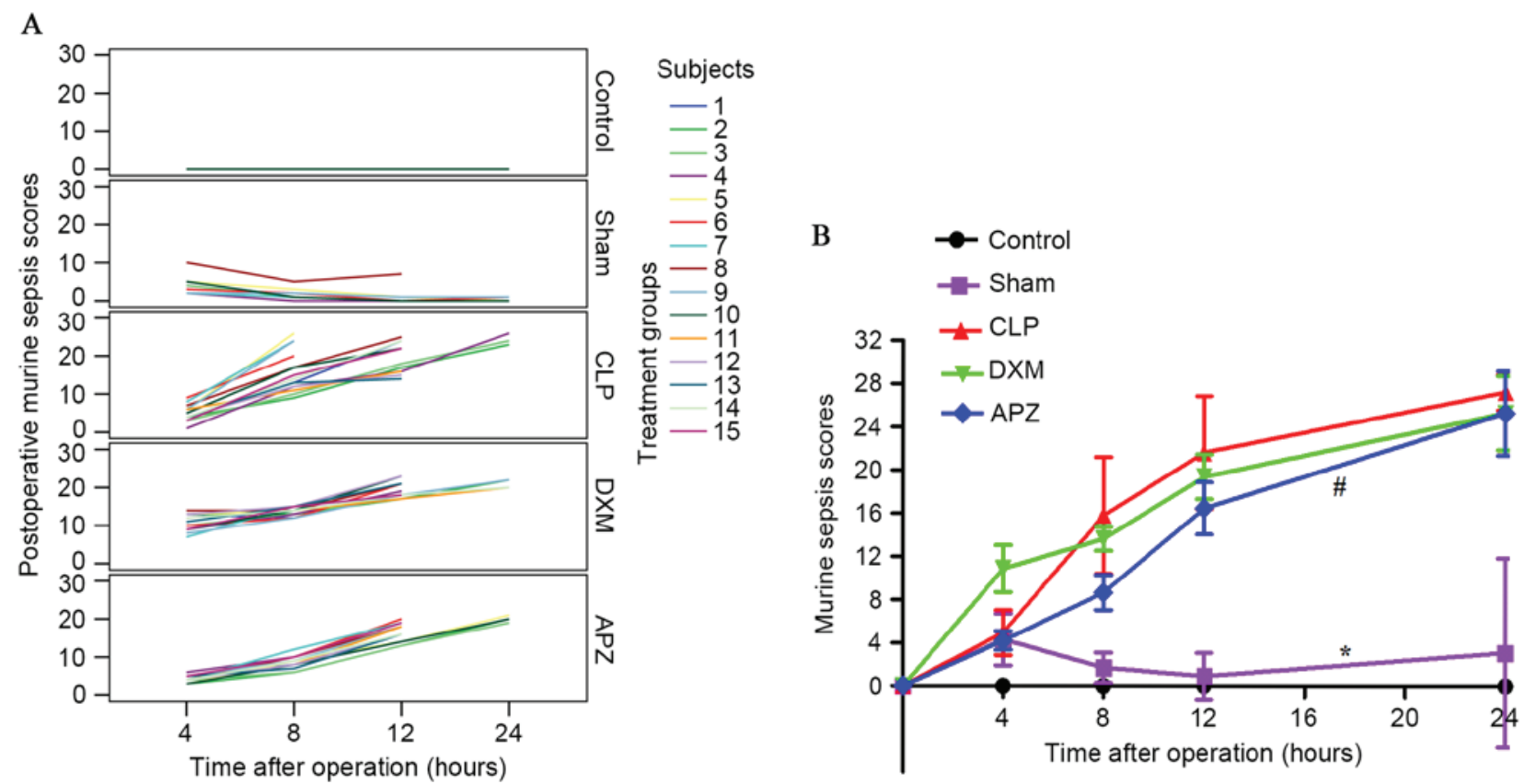

Figure 2. The effect of DXM and its antagonist APZ on the MSS of septic rats induced by CLP. Control, control group (n=10); Sham, sham operation group $(\mathrm{n}=10)$; CLP, CLP group $(\mathrm{n}=15)$; DXM, DXM treatment group $(\mathrm{n}=15)$; APZ, APZ antagonizing group $(\mathrm{n}=15)$. The subjects represent each numbered rat in each group. (A) The MSS scoring at pre-set time-points after operation from individual rats in the different treatment groups without missing data interpolation. (B) Differences of MSS among groups with missing data interpolation were analyzed. " $\mathrm{P}<0.05$, vs. control and CLP groups, respectively; $\mathrm{P}<0.05$, vs. DXM group. DXM, dexmedetomidine; APZ, atepamezole; MSS, murine sepsis scores; CLP, cecal ligation and puncture.

progressed, which was effectively antagonized by APZ. Fig. 3B demonstrates the differences of anal temperature among groups with missing data interpolation. Normal anal temperatures fluctuated between $37.0-38.0^{\circ} \mathrm{C}$ and peaked at $12 \mathrm{~h}$ after surgery (corresponding to $22: 00 \mathrm{~h}$ ). CLP surgery caused significant fever and hypothermia at different stages of sepsis compared with the Sham group, and severely disturbed the circadian rhythm, which was significantly inhibited by DXM pretreatment. APZ significantly antagonized the effect of DXM.

Histological changes of lung tissues. As demonstrated in Fig. 4, the histological changes due to procedures of tissue harvesting were minimal (Fig. 4A and F). Sham surgery caused minimal but significant lung injury compared with the Control group (Fig. 4B and F). CLP surgery induced mild to moderate injury (Fig. 4C and F), which was significantly reduced by DXM pretreatment to a minimal level (Fig. 4D and F). APZ antagonized the beneficial effect of DXM (Fig. 4E and F).

Western blot analysis for caveolin-1 expression in lung. In order to identify whether DXM is able to affect caveolin-1 expression, the expression of caveolin-1 in lung tissues was examined. Fig. 5A demonstrates the caveolin- 1 and $\beta$-actin bands determined by western blotting in different groups at pre-set time-points. Fig. 5B demonstrates the diurnal expression mode of caveolin-1 in the Control group. As indicated in Fig. 5A and B, rats in the Control group expressed a stable 
A

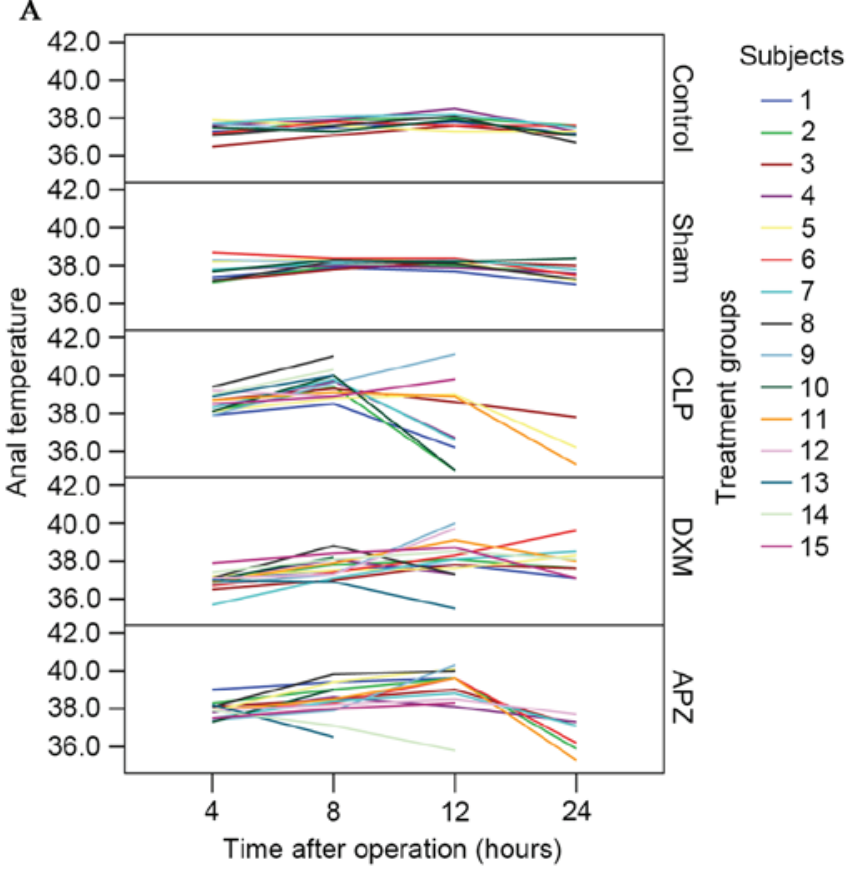

B

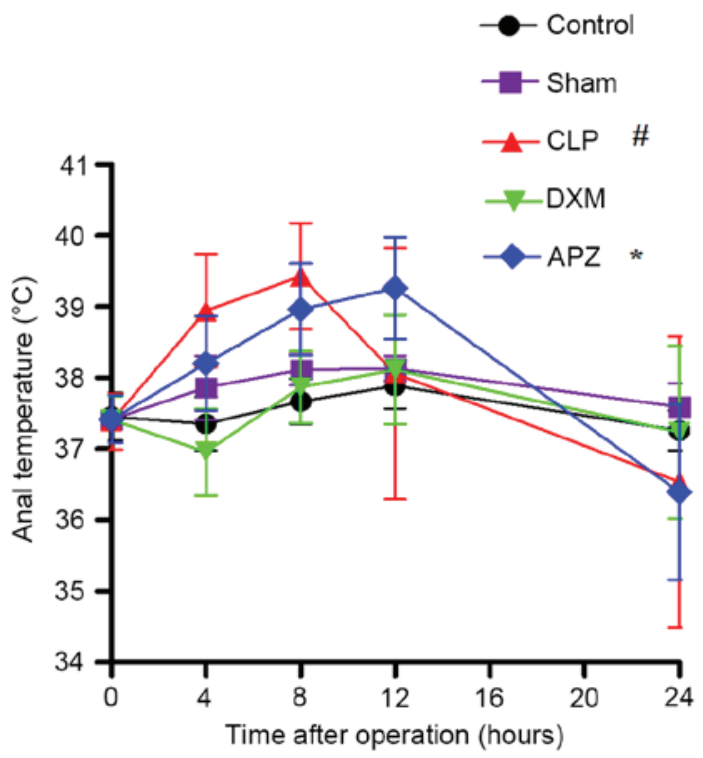

Figure 3. The effect of DXM and its antagonist APZ on temperature of rats with CLP-induced sepsis. Control, control group (n=10); Sham, sham operation group ( $\mathrm{n}=10)$; CLP, CLP operation group ( $\mathrm{n}=15)$; DXM, DXM treatment group $(\mathrm{n}=15)$; APZ, APZ antagonizing group ( $\mathrm{n}=15)$. The subjects represent each numbered rat in each group. (A) The anal temperature $\left({ }^{\circ} \mathrm{C}\right)$ of individual rats at pre-set time-points after operation from different treatment groups without missing data interpolation. (B) Differences of anal temperature among groups with missing data interpolation were analyzed. ${ }^{~} \mathrm{P}<0.05$, vs. Sham and DEX groups; "P<0.05, vs. DXM group. DXM, dexmedetomidine; APZ, atepamezole; CLP, cecal ligation and puncture.
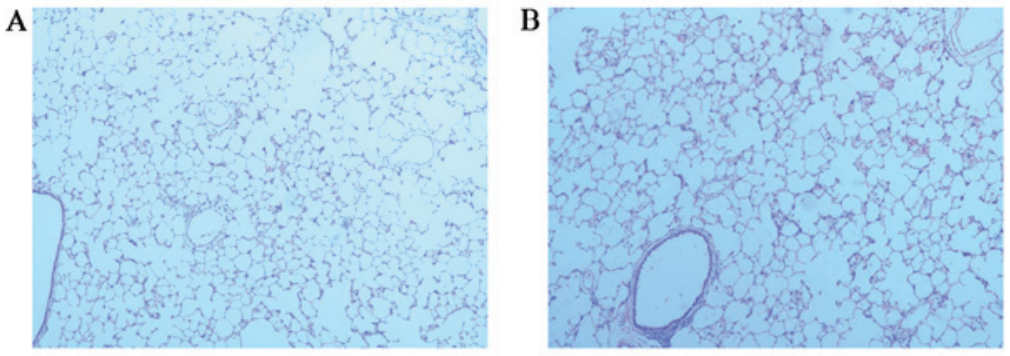

D

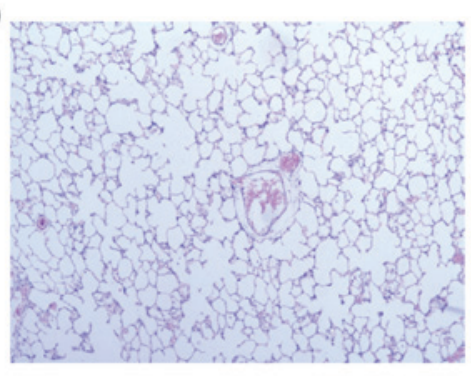

$\mathrm{E}$

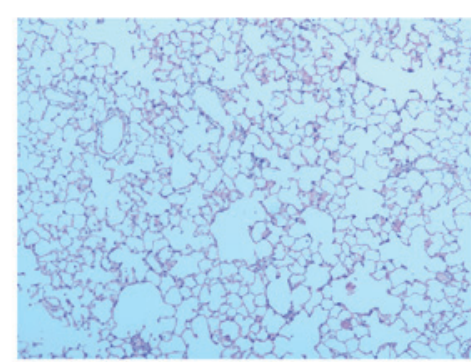

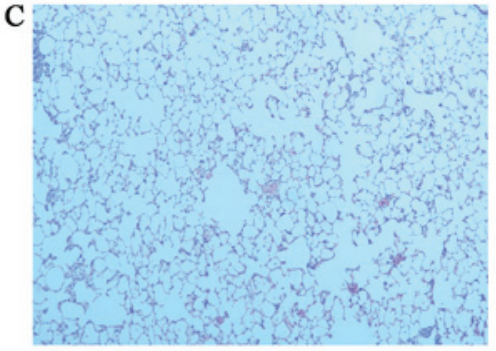

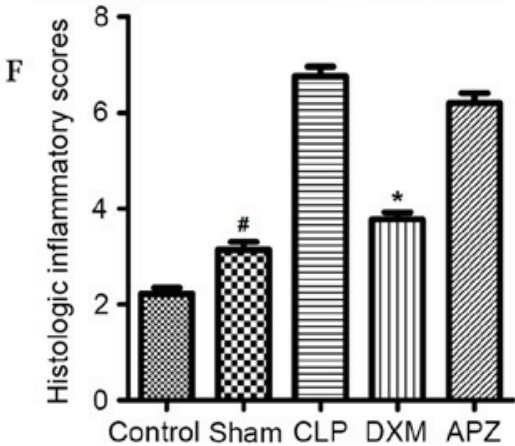

Figure 4. Histological changes of rat lung tissues stained with hematoxylin and eosin (A) Control group (Control); (B) sham operation group (Sham); (C) CLP operation group (CLP); (D) DXM group (DXM); (E) APZ antagonizing group (APZ). Magnification, x4. (F) The histological changes of lung tissue presented in A-E were magnified (magnification, $\mathrm{x} 40$ ) and scored and the data were presented as the mean \pm standard error. The CLP and APZ groups demonstrated mild to moderate injury, whilst Sham and DXM groups demonstrated minimal to mild injury. ${ }^{\#} \mathrm{P}<0.01$, vs. Control and CLP group. ${ }^{*}<0.001$, vs. CLP and APZ group. CLP, cecal ligation and puncture; DXM, dexmedetomidine; APZ, atepamezole.

amount of caveolin-1 with peak expression at the 8-h time-point (corresponding to $\sim$ 4:00 p.m.), and the lowest expression at preand 24-h time-point (corresponding to $~ 8: 00$ a.m.). Fig. 5C indicates the average level of caveolin-1 expression during one day in different treatment groups. Fig. 5D demonstrates the difference of caveolin-1 expression in different treatment groups. As indicated in Fig. 5A, C and D, caveolin-1 expression fluctuated in operated rats, with the exception of the DXM and APZ pretreated groups. DXM significantly upregulated the expression which was markedly inhibited by CLP surgery 


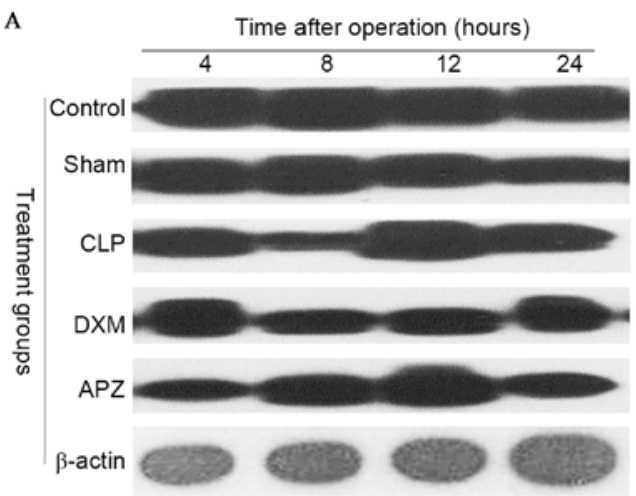

B
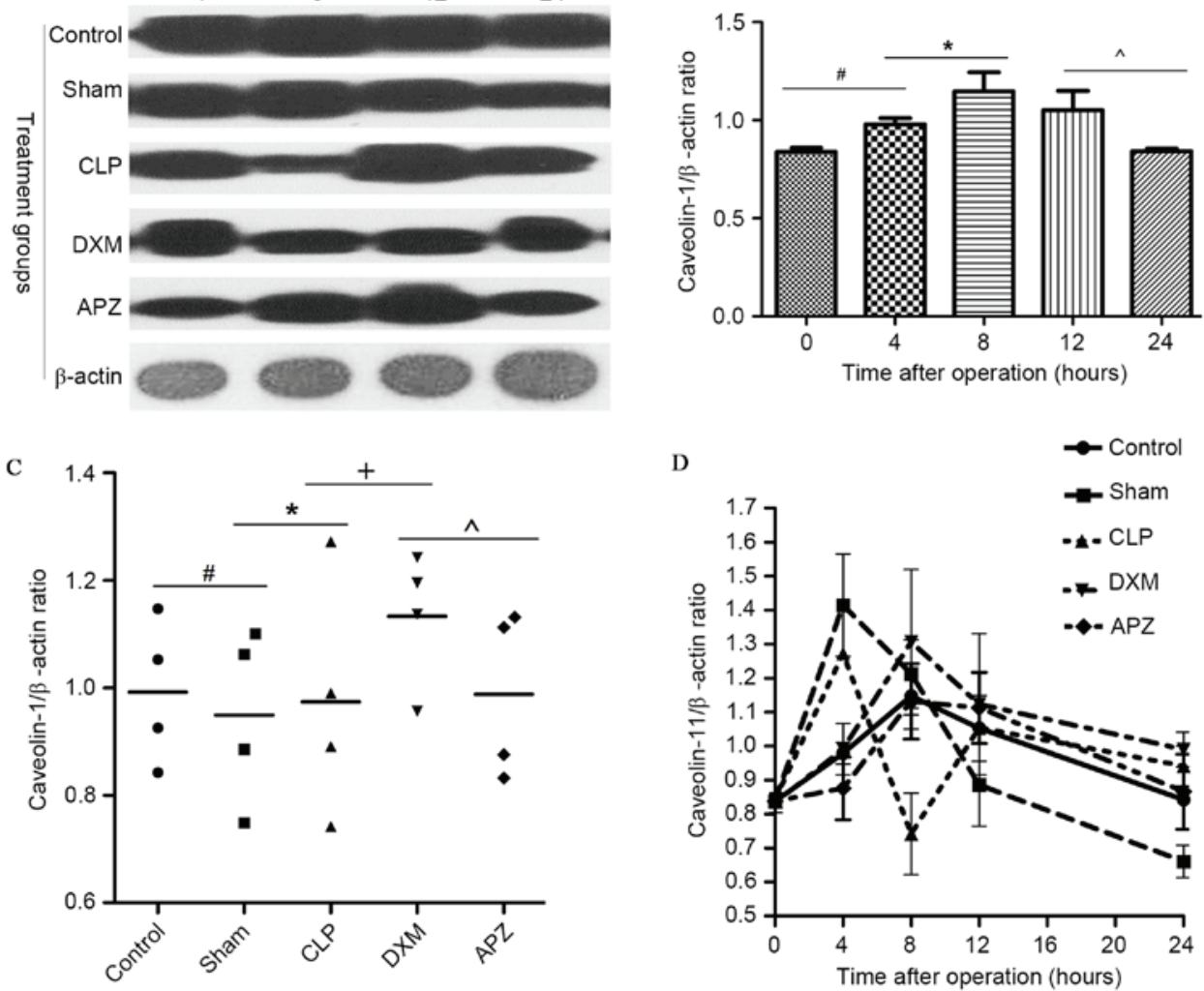

Figure 5. The effect of DXM and its antagonist APZ on caveolin-1 expression in lung tissues of rats with sepsis determined by western blotting. Control, control group ( $\mathrm{n}=10)$; Sham, sham operation group ( $\mathrm{n}=10)$; CLP, CLP operation group ( $\mathrm{n}=15)$; DXM, DXM pretreatment group ( $\mathrm{n}=15)$; APZ, APZ antagonizing group $(\mathrm{n}=15)$. $\beta$-actin was used as the internal standard protein. The grey value of protein band was read by Image $\mathbf{J} 2 \mathrm{X}$ and presented as the mean \pm standard deviation. (A) The caveolin- 1 and $\beta$-actin expression determined by western blotting in different groups at pre-set time-points. (B) The diurnal expression mode of caveolin-1 in control group, the tissue was harvested at the time corresponding to the pre-set time-points in treated groups. ${ }^{\#, *}, \mathrm{P}<0.05$. (C) The average level of caveolin-1 expression during a day in different treatment groups, ${ }^{\sharp, *,+,} \mathrm{P}<0.05$. (D) The difference of caveolin-1 expression in different treatment groups. DXM, dexmedetomidine; APZ, atepamezole; CLP, cecal ligation and puncture.

$4 \mathrm{~h}$ postoperatively. This effect was partly antagonized by APZ. DXM and APZ pretreatment effectively preserved the diurnal rhythm disturbed by CLP surgery.

\section{Discussion}

DXM was recommended as a sedative for septic patients in the ICU due to its anti-inflammatory effects, however its underlying mechanisms and long-term effects remain unclear. In the present study, it was identified that DXM pretreatment at a single sedative/hypnotic dose (20 $\mu \mathrm{g} / \mathrm{kg}$, i.p.) effectively decreased 24-h mortality and acute lung injury, and inhibited the increase of temperature, however it failed to reduce the increase of blood lactate, glucose and MSS scores. It was demonstrated that DXM can upregulate the expression and partly preserve the diurnal rhythm of caveolin-1 in lung tissues. APZ could partly neutralize the effects of DXM.

DXM pharmaceutically causes a dose-dependent inhibition of sympathetic tone in the central and peripheral nervous system and is used for sedation, analgesia, hypotension and hypothermia, its use resulting in a spontaneous motility decrease and induction of sleep. All these effects can be inhibited by prior or simultaneous delivery of APZ $(23,24)$. The results of the present study agreed with this, even though it appeared that DXM marginally increased MSS scores while APZ decreased the scores. Due to the fact that the MSS scoring system gauged activity, consciousness, response to stimuli and eye opening as criteria, DXM could have increased these scores due to its sedative and analgesic activity, and APZ downgraded the scores through the neutralization of these effects. The present study also identified that DXM slightly decreased blood lactate and increased glucose levels, which can be significantly antagonized by APZ. To the best of our knowledge, stress can induce the rise of blood glucose, and tissue hypoxia can induce lactate generation. DXM may have reduced blood glucose due to the inhibition of stress through sedation and analgesia, however the effect of DXM on blood glucose and insulin secretion was more complex, since it can directly inhibit insulin secretion through activation of $\alpha 2-\mathrm{AR}$ and imidazoline receptors on pancreatic $\beta$ cells (25), but also diminished activation of $\alpha 1-\mathrm{AR}$ and $\beta$-AR through reducing the sympathoadrenal output: The balance of these factors may have resulted in different glucose levels (26). These effects on peripheral sympathetic tone in addition with its central sympatholysis and vagal effect can significantly reduce systemic blood pressure. In addition, DXM was reported to enhance micturition through $\alpha 2 \mathrm{~b}-\mathrm{AR}$ on the proximal tubule endothelia (27), which may further decrease circulatory volume and blood pressure when not under continuous fluid perfusion. These effects may have contributed to the failure of DXM to 
effectively reduce the hypoxia in tissues and the accumulation of lactate, even following the balance of its favorable effect in the preservation of capillary perfusion through attenuation of leukocyte-endothelial interaction and micro-emboli generation (28). Above all, DXM may be a promising sedative and antipyretic in patients with sepsis, and may be promising for clearing blood lactate after efficient fluid resuscitation. However, DXM may be detrimental to patients who survive sepsis, with the exception of the unclear effect on lactate, due to the fact that the effect of central hypothermia and glucose upregulation could bring patients into a chronic infectious or susceptible state (29). This indicates that the long-term effect of DXM on patients with sepsis or critically ill patients who survive should be further studied to assist clinical practice.

DXM was reported to inhibit lung injury and improve survival rate in CLP-induced rats (8), which was in agreement with the results of the present study. These results indicated that the anti-inflammatory action of DXM may be mediated by inhibition of the TLR4/MyD88/NF- $\kappa \mathrm{B}$ signaling pathway. The TLR4-dependent signaling pathway mediates neutrophil sequestration into the lungs and causes secondary lung injury due to endotoxin or hyperinflation (30). Additional studies have implied that the receptors mediate the anti-inflammatory actions of DXM possibly through $\alpha 2-\mathrm{AR}$ and IRs, although one study used APZ as an antagonist in a renal ischemic-reperfusion injury (IRI) rat model (11) and the other utilized yohimbine in a lung IRI rats model (9). It is known that DXM binds to $\alpha 2-\mathrm{AR}$ and specifically activates the Gai subunit, which has been demonstrated to bind with the N-terminus (Residual 82-101) of caveolin-1 prior to being activated, and to translocate away from caveolin-1, concomitantly releasing G $\beta \gamma$ subunits after activation (31). Studies have also demonstrated that Src Tyrosine kinases, H-ras and eNOS share the same binding domain of caveolin-1 (32), the G $\beta \gamma$ complex induces activation of Src after release (33) and the later phosphorylated caveolin-1 at Tyr14 induces interaction with TLR4 and mediates MyD88-dependent signaling (15). Deficiency of caveolin-1 would reduce TLR4 signaling (34). However, knockout studies have identified that caveolin-1 protects against sepsis by modulating inflammatory responses, alleviating bacterial burden and suppressing thymocyte apoptosis in rats with CLP-induced sepsis (35). In brief, caveolin-1 may biphasicly regulate the TLR4-dependent inflammatory responses.

In the present study, the expression of caveolin-1 in the lung was determined and it was identified that DXM can effectively inhibit the increase of caveolin-1 at $4 \mathrm{~h}$ and the decrease at $8 \mathrm{~h}$ postoperatively induced by CLP surgery, and promote the average expression amount. APZ only partly antagonized the upregulation effect of DXM. This implied that DXM could exert influence on the TLR4 pathway-mediated inflammatory responses and the promote sepsis survival rate through regulation of caveolin-1 expression, however the exact receptors and downstream molecular events require additional investigation.

In conclusion, preemptive clinical sedative doses of DXM may upregulate the expression of caveolin-1 downregulated by sepsis, and may contribute to the inhibition of inflammatory pathways such as the TLR4-mediated pathways. Furthermore, DXM may favor the improvement of short-term outcome by the regulation of other metabolic pathways.

\section{Acknowledgements}

The present study was supported by the Provincial Natural Science Foundation of Hunan, China (grant nos. 2013FJ4082 and 2015SK2085).

\section{References}

1. Dellinger RP, Levy MM, Rhodes A, Annane D, Gerlach H, Opal SM, Sevransky JE, Sprung CL, Douglas IS, Jaeschke R, et al: Surviving sepsis campaign: International guidelines for management of severe sepsis and septic shock, 2012. Intensive Care Medicine 39: 165-228, 2013.

2. Xiao H, Siddiqui J and Remick DG: Mechanisms of mortality in early and late sepsis. Infect Immun 74: 5227-5235, 2006.

3. Ernsberger P, Giuliano R, Willette RN and Reis DJ: Role of imidazole receptors in the vasodepressor response to clonidine analogs in the rostral ventrolateral medulla. J Pharmacol Exp Ther 253: 408-418, 1990.

4. Barr J, Fraser GL, Puntillo K, Ely EW, Gélinas C, Dasta JF, Davidson JE, Devlin JW, Kress JP, Joffe AM, et al: Clinical practice guidelines for the management of pain, agitation, and delirium in adult patients in the intensive care unit. Crit. Care Med 41: 263-306, 2013.

5. Pandharipande PP, Sanders RD, Girard TD, McGrane S, Thompson JL, Shintani AK, Herr DL, Maze M and Ely EW; MENDS investigators: Effect of dexmedetomidine versus lorazepam on outcome in patients with sepsis: An apriori-designed analysis of the MENDS randomized controlled trial. Crit Care 14: R38, 2010.

6. Memiş D, Hekimoğlu S, Vatan I, Yandim T, Yüksel M and Süt N: Effects of midazolam and dexmedetomidine on inflammatory responses and gastric intramucosal $\mathrm{pH}$ to sepsis, in critically ill patients. Br J Anaesth 98: 550-552, 2007.

7. Fraser GL, Devlin JW, Worby CP, Alhazzani W, Barr J, Dasta JF, Kress JP, Davidson JE and Spencer FA: Benzodiazepine versus nonbenzodiazepine-based sedation for mechanically ventilated, critically ill adults: A systematic review and meta-analysis of randomized trials. Crit Care Med 41 (Suppl 1): S30-S38, 2013.

8. Wu Y, Liu Y, Huang H, Zhu Y, Zhang Y, Lu F, Zhou C, Huang L, Li X and Zhou C: Dexmedetomidine inhibits inflammatory reaction in lung tissues of septic rats by suppressing TLR4/NF-kB pathway. Mediators Inflamm 2013: 562154, 2013.

9. Jiang L, Li L, Shen J, Qi Z and Guo L: Effect of dexmedetomidine on lung ischemia-reperfusion injury. Mol Med Rep 9: 419-426, 2014.

10. Snapir A, Talke P, Posti J, Huiku M, Kentala E and Scheinin M: Effects of nitric oxide synthase inhibition on dexmedetomidine-induced vasoconstriction in healthy human volunteers. $\mathrm{Br}$ J Anaesth 102: 38-46, 2009.

11. Si Y, Bao H, Han L, Shi H, Zhang Y, Xu L, Liu C, Wang J, Yang X, Vohra A and Ma D: Dexmedetomidine protects against renal ischemia and reperfusion injury by inhibiting the JAK/STAT signaling activation. J Transl Med 11: 141, 2013.

12. Liu L, Brown D, McKee M, Lebrasseur NK, Yang D, Albrecht KH, Ravid K and Pilch PF: Deletion of Cavin/PTRF causes global loss of caveolae, dislipidemia, and glucose intolerance. Cell Metab 8: 310-317, 2008.

13. Fu Y, Moore XL, Lee MK, Fernández-Rojo MA, Parat MO, Parton RG, Meikle PJ, Sviridov D and Chin-Dusting JP: Caveolin-1 plays a critical role in the differentiation of monocytes into macrophages. Arterioscler Thromb Vasc Biol 32: e117-e125, 2012.

14. Feng H, Guo L, Song Z, Gao H, Wang D, Fu W, Han J, Li Z, Huang B and Li XA: Caveolin-1 protects against sepsis by modulating inflammatory response, alleviating bacterial burden, and supressing thymocyte apoptosis. J Biol Chem 285: 25154-25160, 2010.

15. Jiao H, Zhang Y, Yan Z, Wang ZG, Liu G, Minshall RD, Malik AB and Hu G: Caveolin-1 Tyr14 phosphorylation induces interaction with TLR4 in endothelial cells and mediates MyD88-dependent signaling and sepsis-induced lung inflammation. J Immunol 191: 6191-6199, 2013.

16. Calizo RC and Scarlata S: A role for G-proteins in directing G-protein-coupled receptor-caveolae localization. Biochemistry 51: 9513-9523, 2012. 
17. D'Alessio A, Kluger MS, Li JH, Al-Lamki R, Bradley JR and Pober JS: Targeting of tumor necrosis factor receptor 1 to low density plasma membrane domains in human endothelial cells. J Biol Chem 285: 23868-23879, 2010.

18. Chen Z, Bakhshi FR, Shajahan AN, Sharma T, Mao M, Trane A, Bernatchez P, van Nieuw Amerongen GP, Bonini MG, Skidgel RA, et al: Nitric oxide-dependent Src activation and resultant caveolin-1 phosphorylation promote eNOS/caveolin-1 binding and eNOS inhibition. Mol Biol Cell 23, 1388-1398, 2012

19. Vetterkind S, Poythress RH, Lin QQ and Morgan KG: Hierarchical scaffolding of an ERK1/2 activation pathway. Cell Commun Signal 11: 65, 2013.

20. Banquet S, Delannoy E, Agouni A, Dessy C, Lacomme S, Hubert F, Richard V, Muller B and Leblais V: Role of Gi/o-Src kinase-PI3K/Akt pathway and caveolin-1 in $\beta_{2}$-adrenoceptor coupling to endothelial NO synthase in mouse pulmonary artery. Cell Signal 23: 1136-1143, 2011.

21. Toscano MG, Ganea D and Gamero AM: cecal ligation puncture procedure. J Vis Exp: 2860, 2011.

22. Shrum B, Anantha RV, Xu SX, Donnelly M, Haeryfar SM, McCormick JK and Mele T: A robust scoring system to evaluate sepsis severity in an animal model. BMC Res Notes 7: 233, 2014.

23. Virtanen R: Pharmacological profiles of medetomidine and its antagonist, atipamezole. Acta Vet Scand Suppl 85: 29-37, 1989.

24. Madden CJ, Tupone D, Cano G and Morrison SF: $\alpha 2$ adrenergic receptor-mediated inhibition of thermogenesis. J Neurosci 33: 2017-2028, 2013.

25. Takahashi T, Kawano T, Eguchi S, Chi $\mathrm{H}$, Iwata $\mathrm{H}$ and Yokoyama M: Effects of dexmedetomidine on insulin secretion from rat pancreatic $\beta$ cells. J Anesth 29: 396-402, 2015.

26. Restitutti F, Raekallio M, Vainionpää M, Kuusela E and Vainio O: Plasma glucose, insulin, free fatty acids, lactate and cortisol concentrations in dexmedetomidine-sedated dogs with or without MK467: A peripheral $\alpha$-2adrenoceptor antagonist. Vet J 193: 481-485, 2012.
27. Cussac D, Schaak S, Gales C, Flordellis C, Denis C and Paris H: alpha(2B)-adrenergic receptors activate MAPK and modulate proliferation of primary cultured proximal tubule cells. Am J Physiol Renal Physiol 282: F943-F952, 2002.

28. Miranda ML, Balarini MM and Bouskela E: Dexmedetomidine attenuates the microcirculatory derangements evoked by experimental sepsis. Anesthesiology 122: 619-630, 2015.

29. Drewry AM, Fuller BM, Skrupky LP and Hotchkiss RS: The presence of hypothermia within 24 hours of sepsis diagnosis predicts persistent lymphopenia. Crit Care Med 43: 1165-1196, 2015.

30. Hu G, Malik AB and Minshall RD: Toll-like receptor 4 mediates neutrophil sequestration and lung injury induced by endotoxin and hyperinflation. Crit care med 38: 194-201, 2010.

31. Li S, Okamoto T, Chun M, Sargiacomo M, Casanova JE, Hansen SH, Nishimoto I and Lisanti M: Evidence for a regulated interaction between heterotrimeric $\mathrm{G}$ proteins and caveolin. J Biol Chem 270: 15693-15701, 1995.

32. Li S, Couet J and Lisanti MP: Src tyrosine kinases, Galpha subunits, and $\mathrm{H}-\mathrm{Ras}$ share a common membrane-anchored scaffolding protein, caveolin. Caveolin binding negatively regulates the auto-activation of Src tyrosine kinases. J Biol Chem 271: 29182-29190, 1996.

33. Shajahan AN, Tirupathi $\mathrm{C}$ and Minshall RD: Gbetagamma activation of Src induces caveolae-mediated endocytosis in endothelial cells. J Biol Chem 279: 48055-48062, 2004.

34. Mirza MK, Yuan J, Gao XP, Garrean S, Brovkovych V, Malik AB, Tiruppathi C and Zhao YY: Caveolin-1 deficiency dampens Toll-like receptor 4 signaling through eNOS activation. Am J Pathol 176: 2344-2351, 2010.

35. Feng H, Guo L, Song Z, Gao H, Wang D, Fu W, Han J, Li Z, Huang B and Li XA: caveolin-1 protects against sepsis by modulating inflammatory response, alleviating bacterial burden, and supressing thymocyte apoptosis. J Biol Chem 285: 25154-25160, 2010. 